Research Article

\title{
DFT Calculations for the HONO Elimination Process of CL-20 Conformers
}

\author{
Lin-Lin Liu $\mathbb{D}^{\mathbb{D}}$, Tong-Yong Zhang, and Song-Qi Hu \\ Science and Technology on Combustion, Internal Flow and Thermo-Structure Laboratory, Northwestern Polytechnical University, \\ Xi'an 710072, China \\ Correspondence should be addressed to Lin-Lin Liu; 1l1@nwpu.edu.cn
}

Received 26 October 2021; Revised 2 December 2021; Accepted 8 December 2021; Published 22 December 2021

Academic Editor: Marcelino Maneiro

Copyright (C) 2021 Lin-Lin Liu et al. This is an open access article distributed under the Creative Commons Attribution License, which permits unrestricted use, distribution, and reproduction in any medium, provided the original work is properly cited.

The HONO elimination process is regarded to be an important initial decomposition process of energetic nitramines. Four CL-20 conformers based on the $\varepsilon$-CL-20 were obtained by the optimization at the $\mathrm{m} 062 \mathrm{x} / \mathrm{cc}$-pvtz level in this study, and the Transition State (TS) and Intrinsic Reaction Coordinate (IRC) calculations were carried out at the same level. In addition, the rate coefficients and activation energy of the HONO elimination process were evaluated using conventional transition state theory (TST) and canonical variational transition state theory (CVT) with Eckart and small-curvature tunneling (SCT) methods to correct the transmission coefficients for the quantum tunneling effect. The calculation results have shown that the HONO elimination process concerning the nitro groups located on six numbered rings is the hardest to happen, and it seems that the longer distance between nitro groups and the adjacent hydrogen atom would result in the higher barrier energy; the HONO elimination process is most likely to happen for the axial positioning of nitro groups located on five numbered rings and most unlikely to happen for the ones located on six numbered rings; CL-20 II and CL-20 IV conformers are the most unstable one and most stable one concerning the reaction difficulty of the HONO elimination process.

\section{Introduction}

2,4,6,8,10,12-Hexanitro-2,4,6,8,10,12- hexaazaisowurtzitane (CL-20 or HNIW), a promising heterocyclic energetic nitramine, like 1,3,5-trinitroperhydro-1,3,5-triazine (RDX) and octahydro-1,3,5,7-tetranitro-1,3,5,7-tetrazocine (HMX), contains the characteristic $\mathrm{N}-\mathrm{NO}_{2}$ functional groups. Compared to the other energetic nitramines, there are six $\mathrm{N}-\mathrm{NO}_{2}$ groups in a CL-20 molecule which results in an increase in both density and heat of formation. In addition, CL-20 has excellent chemical stability, moderate sensitivity, and smokeless combustion products [1]. Therefore, it is considered to be an ideal ingredient of the advanced solid propellants, gun power, explosives, and gasgenerating agents $[2,3]$.

The decomposition of CL-20 could provide a lot of valuable information for the ignition, combustion, and explosion mechanisms of the corresponding energetic materials [4]. There are generally two pathways of thermal decomposition mechanisms of energetic nitramines, i.e., unimolecular reactions and multimolecular reactions.

The unimolecular pathway mainly refers to the initiated gas-phase thermolysis, photolysis, and orionization in molecule beams, while the complex multimolecular reactions in the condensed phase are usually involved for the multimolecular process. In fact, the decomposition of energetic nitramines is quite complicated to investigate even for the unimolecular reactions of simpler RDX and HMX because of the hundreds of involving elementary reactions [5-7].

The initial step is an important issue concerning the decomposition of energetic nitramines, and it is believed that $\mathrm{NO}_{2}$ fission (also called $\mathrm{N}-\mathrm{N}$ bond dissociation), HONO elimination, concerted ring scission, and ring dissociation along the $\mathrm{C}-\mathrm{N}$ bond are the most possible pathways for the initial step of RDX and HMX molecules [8-10]. There have been several studies about the decomposition of CL-20, whereas most of them focus on the condensed phase 
which is usually associated with the complex multimolecular process $[11,12]$.

The individual steps are the key issues for the decomposition mechanisms, and they are generally difficult to be investigated, especially by the experiments because of the high reaction rate and heat release. $\mathrm{Ab}$ initio calculations provide an important method to obtain the detailed decomposition steps of energetic materials, and there are several studies concerning the CL-20 molecule [13]. For example, some computational results show that the $\mathrm{NO}_{2}$ fission process of CL-20 is much different from that of $\mathrm{RDX}$ and HMX [14].

The purposes of this paper are to investigate the pathway of the HONO elimination process for CL-20 conformers and obtain the reaction parameters under different temperatures. The results may provide valuable information for the decomposition and combustion mechanisms of CL-20.

\section{Computation}

There are two five-member rings and a six-member ring in the caged CL-20 molecule, and the six nitro groups are attached to each of the six bridging nitrogen atoms in the cage. CL-20 has five crystal polymorphs, denoted as $\alpha$-, $\beta$-, $\gamma_{-}, \varepsilon-$, and $\zeta$-CL-20 [15-17], and four stable CL-20 conformers based on these polymorphs were used in this paper (Figure 1). The transition states (TS) of the HONO elimination reactions were optimized with the verification of the vibrational analyses by solely an imaginary frequency. Starting from the TS, the intrinsic reaction coordinates (IRCs) were searched and confirmed to connect correctly to the associated reactants and products. The structural optimizations, energy profiles, IRC, and frequency calculations were expedited with Gaussian 09 program [18] at the m062x/ cc-pvtz level.

The rate coefficients of the reactions at a temperature range of $400 \mathrm{~K} \sim 2500 \mathrm{~K}$ were evaluated by conventional transition theory (TST) and canonical variation transition state theory (CVT) methods by using VKLab package [19], and the one-dimensional Eckart formula [20] and smallcurvature tunneling correction [21] were employed to correct the transmission coefficients for the quantum tunneling effect. The Eckart method accounts for the barrier height by fitting an Eckart potential, and the Schrödinger equation accounting for tunneling can be solved exactly. Small-curvature tunneling correction is a semiclassical multidimensional method to provide accurate transmission coefficients.

\section{Results and Discussions}

3.1. Structure of CL-20 Conformers. The four optimized conformers of $\zeta$-CL-20 (I), $\alpha$-CL-20 and $\gamma$-CL-20 (II), $\varepsilon$-CL20 (III), and $\beta$-CL-20 (IV) are presented in Figure 1, which belong to the $C_{2 \mathrm{~V}}, C_{1}, C_{1}$, and $C_{2}$ point group, respectively [22].

Heterocyclic energetic nitramine molecules are usually identified according to axial (A) or pseudoequatorial positioning (E) of the nitro groups about the ring [23]. There are six nitro groups in the CL-20 conformer, and four of them are located on five numbered rings. There are four, three, and two nitro groups occupying axial positions of the five numbered rings for I, II, III, and IV conformers, and then, these conformers can be labeled by the nitro group position relative to the five numbered ring atoms. Therefore, there are mainly eleven different nitro groups according to the symmetry of the conformers, resulting in the same number of HONO elimination process (shown in Figure 1) which should be studied in this paper.

3.2. Reaction Path of HONO Elimination Processes. IRC calculation results indicate that each HONO elimination reactions have one transition state, and the triplet potential energy surface (PES) of R11 and R12 is shown in Figure 2 which is similar with the other reactions except for the energy level and molecule structure differences.

IRC results reveal the existence of transition states for all the reactions, and the energy of transition states is higher than that of the corresponding reactants and products. Therefore, the rate coefficients could be calculated by using TST and CVT methods. In addition, it seems that the reaction rate of R11 is lower than that of R12 because of the higher reaction barrier between the transition state and reactant.

The results presented in Table 1 show that the HONO elimination processes are endothermic and nonspontaneous under room temperature due to the positive enthalpy and Gibbs free energy of reaction. HONO elimination is difficult to appear under lower temperature owing to the much higher energy barrier. The barrier energy shown in Table 1 also indicates that HONO elimination for the nitro groups located on six numbered rings is the hardest to happen, and the pseudoequatorial positioning of nitro groups is secondary in most cases. It seems that the barrier energy mainly depends on the distance between nitro groups and the adjacent hydrogen atom $\left(d_{\mathrm{NH}}\right)$, and the large distance would result in the higher barrier energy [24, 25]. The distance between the nitro groups located on the five numbered rings and the adjacent hydrogen atom is significantly affected by the conformer structure, i.e., relative position of pseudoequatorial and axial positioning of nitro groups, which results in the barrier energy difference. Therefore, the CL-20 IV conformer with good symmetry of nitro groups will result in the similar $d_{\mathrm{NH}}$ and, thus, much closer energy barrier of R42 and R43.

\subsection{Rate Coefficients of the HONO Elimination Processes.} There are three types of nitro groups for the CL-20 conformers investigated in this paper, and the rate coefficient calculation results of the CL-20 II conformer (Figures 3 5) are employed to show the rate coefficient differences firstly.

Figure 3 shows that TST and CVT calculations could obtain the much similar rate coefficients under lower temperature, and the results are almost unaffected by the quantum tunneling effect. However, the rate coefficients obtained by the TST method are higher than those of the CVT method when the temperature is higher than $900 \mathrm{~K}$, 


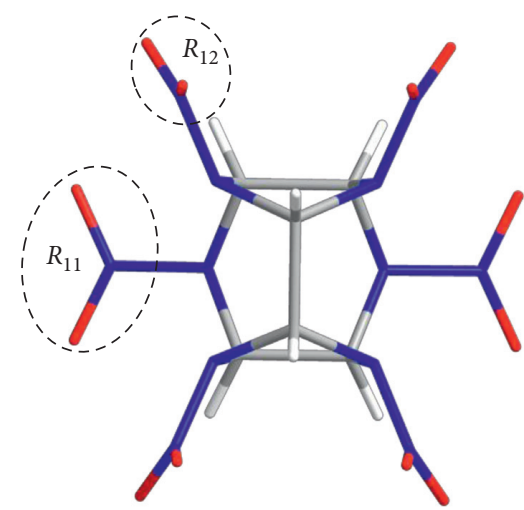

(I)

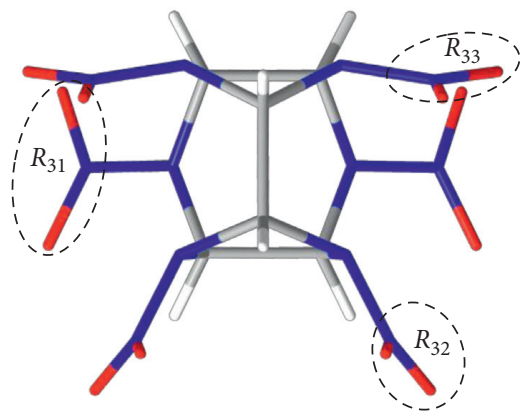

(III)

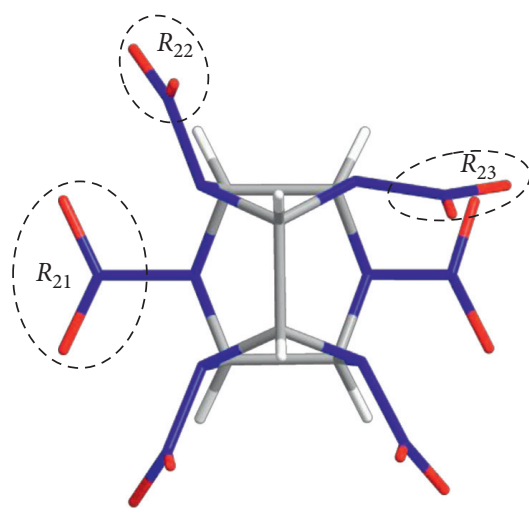

(II)

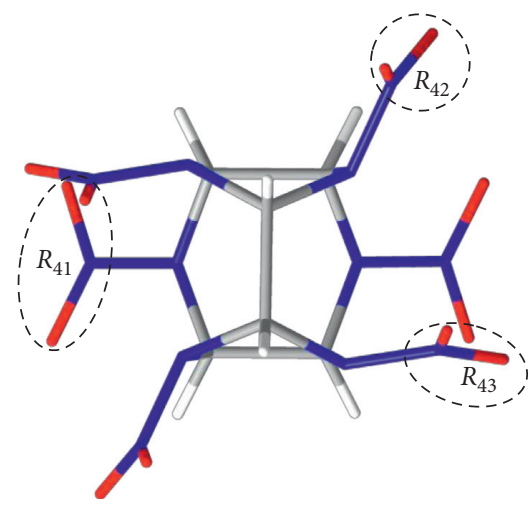

(IV)

FIgure 1: Optimized structures and HONO elimination pathway of CL-20 conformers.

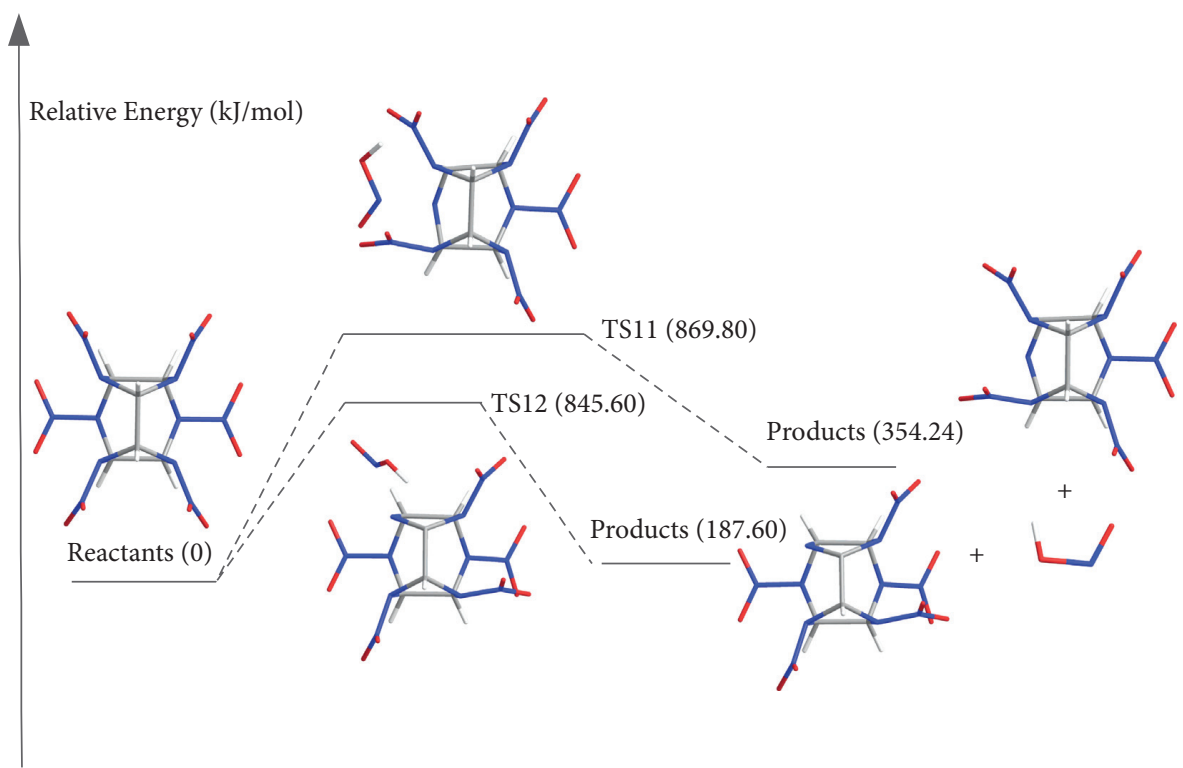

FIGURE 2: IRC calculations of R11 and R12 reactions.

and the higher the temperature, the more obvious the difference between the results. This difference also reveals that the reaction paths of some molecule may deviate from the MEP which is considered by the CVT method. Therefore, the CVT method should be employed under higher temperature to get the more accurate rate coefficients.
Figure 4 indicates that there is an obvious difference for the rate coefficients obtained by TST and CVT methods, and the results are affected significantly by the quantum tunneling effect above $400 \mathrm{~K}$ when the CVT method was employed. Therefore, it is necessary to use the CVT method involved in the tunneling effect to predict the coefficients of R22. 
TABLE 1: Some information of the reactions.

\begin{tabular}{lccc}
\hline Reaction & Energy barrier $(\mathrm{kJ} / \mathrm{mol})$ & $\triangle H(\mathrm{~kJ} / \mathrm{mol})$ & $\triangle G(\mathrm{~kJ} / \mathrm{mol})$ \\
\hline R11 & 869.80 & 195.92 & 143.67 \\
$\mathrm{R} 12$ & 845.60 & 162.35 & 112.16 \\
$\mathrm{R} 21$ & 281.64 & 229.69 & 171.94 \\
$\mathrm{R} 22$ & 238.47 & 136.05 & 81.99 \\
$\mathrm{R} 23$ & 274.22 & 171.52 & 116.95 \\
$\mathrm{R} 31$ & 317.37 & 232.71 & 171.25 \\
$\mathrm{R} 32$ & 240.31 & 108.07 & 53.35 \\
$\mathrm{R} 33$ & 348.49 & 177.13 & 120.63 \\
$\mathrm{R} 41$ & 291.08 & 238.42 & 178.61 \\
$\mathrm{R} 42$ & 284.76 & 168.99 & 113.88 \\
R43 & 283.65 & 180.25 & 123.63 \\
\hline
\end{tabular}

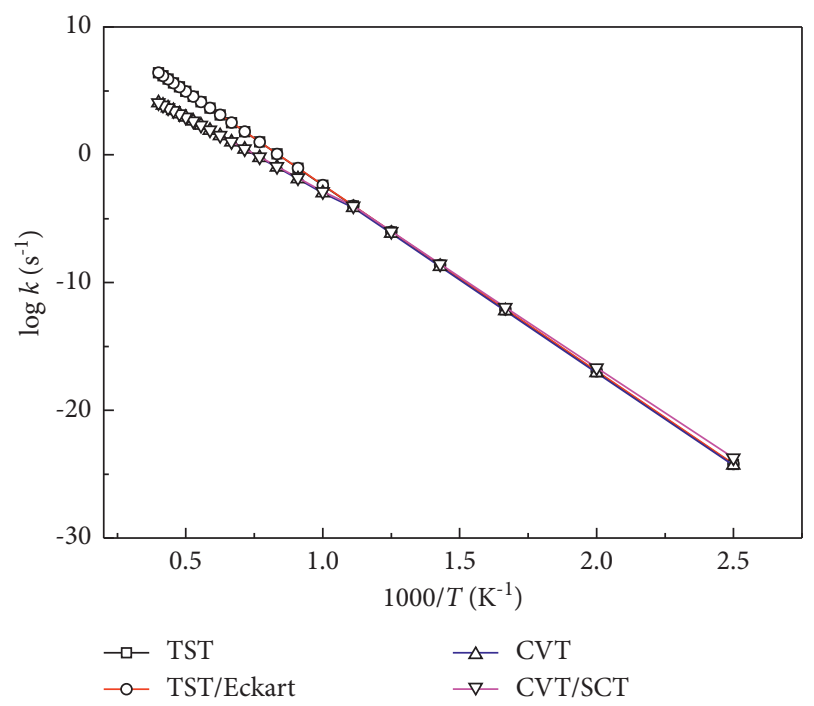

Figure 3: Forward rate coefficient of R21.

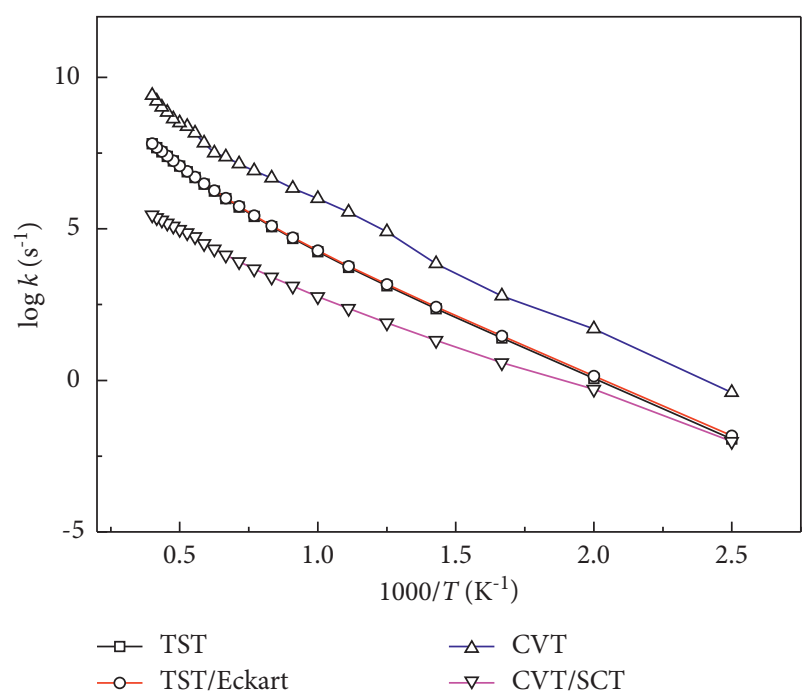

FIgURE 4: Forward rate coefficient of R22.

The rate coefficients are almost unaffected by the quantum tunneling effect which is similar with R21; however, the rate coefficients obtained with the TST method are

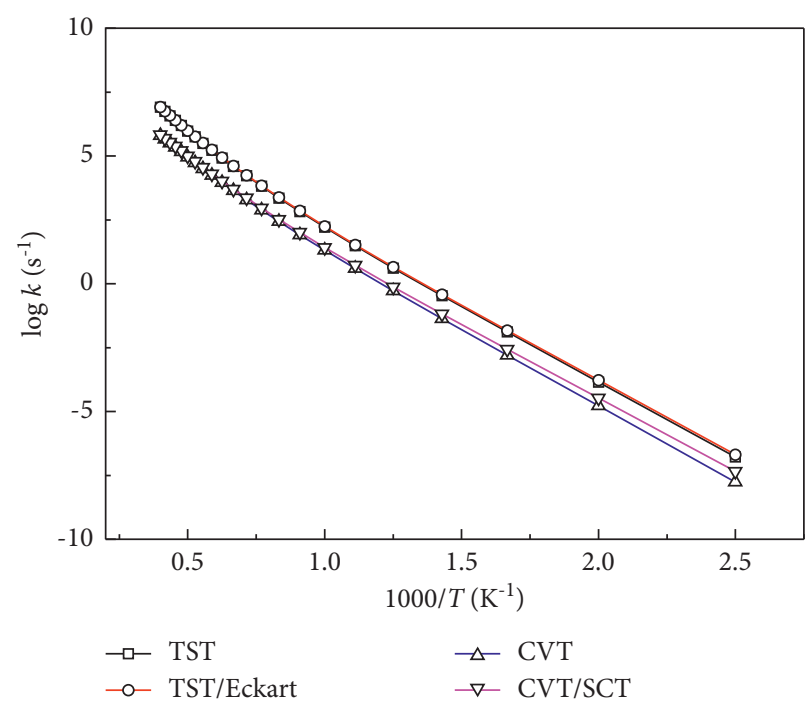

FIGURE 5: Forward rate coefficient of R23.

higher than the ones obtained with the CVT method for the same temperature. Generally, the CVT method is more reliable than the TST method, and the rate coefficients of R23 should be predicted by the CVT method.

In addition, Figures $2 \sim 5$ also suggests the rate coefficient increases obviously with the decrease of temperature, and the reactions generally do not happen when the temperature is less than $700 \mathrm{~K}$ due to the lower reaction rate coefficients. The rate coefficients of the reactions are close under high temperature $\left(10^{4.05} \mathrm{~s}^{-1}, 10^{5.45} \mathrm{~s}^{-1}\right.$, and $10^{5.82} \mathrm{~s}^{-1}$ for R21, R22, and R23 at $2500 \mathrm{~K}$ ); however, there is much difference under low temperature $\left(10^{-16.67} \mathrm{~s}^{-1}, 10^{-1.82} \mathrm{~s}^{-1}\right.$, and $10^{-7.76} \mathrm{~s}^{-1}$ for R21, R22, and R23 at $400 \mathrm{~K}$ ) when the CVT/SCT method was employed. Generally, R22 has the highest rate coefficients and R21 has the lowest rate coefficient which is consistent with the results of energy barriers.

In this study, the two-parameter Arrhenius expression $k=A e^{-\left(E_{a} / R T\right)}$ was used to fitting the reaction rate curves obtained by the CVT/SCT method, and the results are shown in Table 2.

As shown in Table 2, the highest HONO elimination activation energy $\left(E_{a}\right)$ of the nitro groups located on six numbered rings also confirms the difficulty of the reactions for all the CL-20 conformers, and the lowest $E_{a}$ of the axial positioning of nitro groups also means the reactions are the easiest to happen. In addition, the $E_{a}$ of R22 and R23 is $43.14 \mathrm{~kJ} / \mathrm{mol}$ and $92.52 \mathrm{~kJ} / \mathrm{mol}$ which are much lower than the ones of the other reaction, and the HONO elimination process is most likely to happen for the Cl-20 II conformer.

$\mathcal{E}$-CL-20 is the most stable crystal form which corresponds to the CL-20 III conformer; however, the most stable conformer is CL-20 IV when the lowest HONO elimination activation energy for each conformer is compared. Our previous study [26] showed that the activation energy for $\mathrm{NO}_{2}$ fission of CL-20 conformers is in the $171.40 \mathrm{~kJ} /$ mol $219.85 \mathrm{~kJ} / \mathrm{mol}$ range, which is lower than that for HONO elimination obviously $(248.03 \mathrm{~kJ} / \mathrm{mol} 291.05 \mathrm{~kJ} /$ $\mathrm{mol}$ ), and the difference is more obvious for the rate 
TABLE 2: Arrhenius parameters of the elimination reactions.

\begin{tabular}{lcc}
\hline Reaction & $A /\left(\mathrm{s}^{-1}\right)$ & $E_{a} /(\mathrm{kJ} / \mathrm{mol})$ \\
\hline $\mathrm{R} 11$ & $2.17 E+10$ & 289.62 \\
$\mathrm{R} 12$ & $1.74 E+20$ & 204.28 \\
$\mathrm{R} 21$ & $3.94 E+07$ & 294.56 \\
$\mathrm{R} 22$ & $5.18 E+19$ & 43.14 \\
$\mathrm{R} 23$ & $1.98 E+19$ & 92.52 \\
$\mathrm{R} 31$ & $1.82 E+11$ & 307.11 \\
$\mathrm{R} 32$ & $2.26 E+12$ & 222.86 \\
$\mathrm{R} 33$ & $1.10 E+12$ & 283.19 \\
$\mathrm{R} 41$ & $4.29 E+11$ & 291.95 \\
$\mathrm{R} 42$ & $3.74 E+13$ & 248.03 \\
$\mathrm{R} 43$ & $1.09 E+12$ & 282.20 \\
\hline
\end{tabular}

coefficient, meaning the much harder HONO elimination reactions. In fact, although the activation energy of HONO elimination decomposition in CL-20 crystal (104 kJ/mol [27]) is lower than the one in the conformer, the reactions are hard to be observed due to the significant influence of the intermolecular effect. Therefore, the calculation results make more sense to the decomposition mechanisms of the CL-20 conformer in the case of solutions and gas-phase combustion.

\section{Conclusions}

According to the results presented in this paper, the following conclusions can be made:

(1) HONO elimination of the CL-20 conformer has a sole transition state. The activation energy of the reactions is consistent with the corresponding energy barrier, and both of them can be employed to evaluate the reaction difficulty. The CVT method with SCT correcting the transmission coefficients should be used to obtain the more reliable rate coefficients.

(2) The HONO elimination process concerning the nitro groups located on six numbered rings is the hardest to happen, and the pseudoequatorial positioning of nitro groups is secondary in most cases. It seems that the longer distance between nitro groups and the adjacent hydrogen atom $\left(d_{\mathrm{NH}}\right)$ would result in the higher barrier energy.

(3) The HONO elimination process is most likely to happen for the axial positioning of nitro groups located on five numbered rings, followed by the pseudoequatorial ones, and the HONO elimination process is difficult to happen for the nitro groups located on six numbered rings.

(4) The HONO elimination process is easy to carry out for the CL-20 II conformer due to the much lower activity energy, especially for R22 (only $43.14 \mathrm{~kJ} / \mathrm{mol}$ ). The CL20 IV conformer is the most stable one concerning the difficulty of the HONO elimination reaction.

\section{Data Availability}

The calculation data used to support the findings of this study are included within the article.

\section{Conflicts of Interest}

The authors declare no conflicts of interest.

\section{Acknowledgments}

This study was supported by the Natural Science Basic Research Program of Shaanxi Province (Grant no. 606150897031) and Center for High Performance Computing in NPU.

\section{References}

[1] R. L. Simpson, P. A. Urtiew, D. L. Ornellas, G. L. Moody, and D. M. Hoffman, "CL-20 performance exceeds that of HMX and its sensitivity is moderate," Propellants, Explosives, Pyrotechnics, vol. 22, pp. 249-255, 2010.

[2] M. Geetha, U. R. Nair, D. B. Sarwade, G. M. Gore, S. N. Asthana, and H. Singh, "Studies on CL-20: The most powerful high energy material," Journal of Thermal Analysis and Calorimetry, vol. 73, no. 3, pp. 913-922, 2003.

[3] R. Ding, J. Xu, Y. Tao, J. Sun, and M. Lei, "Experimental and theoretical study on the stability of CL-20-based host-guest energetic materials," The Journal of Physical Chemistry A, vol. 124 , no. 31, pp. 6389-6398, 2020.

[4] G. F. Adams and R. W. Shaw, "Chemical reactions in energetic materials," Annual Review of Physical Chemistry, vol. 43, no. 1, pp. 311-340, 1992.

[5] K. V. Meredith, M. L. Gross, and M. W. Beckstead, "Laserinduced ignition modeling of HMX," Combustion and Flame, vol. 162, no. 2, pp. 506-515, 2015.

[6] A. A. Zenin and S. V. Finjakov, "Studying RDX and HMX combustion mechanisms by various experimental techniques," Combustion, Explosion and Shock Waves, vol. 45, no. 5, pp. 559-578, 2009.

[7] R. A. Yetter, F. L. Dryer, M. T. Allen, and J. L. Gatto, "Development of gas-phase reaction mechanisms for nitramine combustion," Journal of Propulsion \& Power, vol. 11, pp. 683-697, 2012.

[8] L.-L. Liu, P.-J. Liu, S.-Q. Hu, and G.-Q. He, “Ab initio calculations of the N-N bond dissociation for the gas-phase RDX and HMX," Scientific Reports, vol. 7, no. 1, Article ID 40630, 2017.

[9] M. J. Swadley and T. Li, "Crystallization force--a density functional theory concept for revealing intermolecular interactions and molecular packing in organic crystals," Journal of Chemical Theory and Computation, vol. 3, 2007.

[10] C. A. Wight and T. R. Botcher, "Thermal decomposition of solid RDX begins with nitrogen-nitrogen bond scission," Journal of the American Chemical Society, vol. 114, no. 21, pp. 8303-8304, 1992.

[11] R. Turcotte, M. Vachon, Q. S. M. Kwok, R. Wang, and D. E. G. Jones, "Thermal study of HNIW (CL-20)," Thermochimica Acta, vol. 433, no. 1-2, pp. 105-115, 2005.

[12] Q.-L. Yan, S. Zeman, Z. W. Song, and J. Malek, "Roman svoboda," Journal of Thermal Analysis and Calorimetry, vol. 112, pp. 837-849, 2013.

[13] S. Okovytyy, Y. Kholod, M. Qasim, H. Fredrickson, and J. Leszczynski, "MM Qasim," The Journal of Physical Chemistry A, vol. 109, p. 2964, 2005.

[14] O. Isayev, L. Gorb, M. Qasim, and J. Leszczynski, "Health \& environmental research online (HERO)," Journal of Physical Chemistry B, vol. 112, pp. 11005-11013, 2008. 
[15] M. F. Foltz, "The thermal stability of the polymorphs of hexanitrohexaazaisowurtzitane, Part I," Propellants, Explosives, Pyrotechnics, vol. 19, pp. 63-69, 1994.

[16] M. F Foltz, L. C. Clifford, F. Garcia, and A. L. Nicholes, "The thermal stability of the polymorphs of hexanitrohexaazaisowurtzitane, part II," Propellants Explosives Pyrotechnics, vol. 19, pp. 19-25, 2010.

[17] T. P. Russell, P. J. Miller, G. J. Piermarini, and S. Block, "Pressure/temperature phase diagram of hexanitrohexaazaisowurtzitane," Journal of Physical Chemistry, vol. 96, pp. 5509-5512, 1992.

[18] M. J. Frisch, G. W. Trucks, H. B. Schlegel et al., "Solvent effects on the [3+2] cycloaddition of 2-furfural oxime and ethyl propiolate: unexpected change in regioselectivity," World Journal of Organic Chemistry, vol. 5, no. 1, pp. 6-10, 2017.

[19] S. Zhang and T.N. Troung, VKLab Version 1.0. University of Utah, Salt Lake City, UT, USA, 2001.

[20] C. Eckart, "The theory and calculation of screening constants," Physical Review, vol. 35, pp. 1303-1309, 1930.

[21] W. P. Hu, Y. P. Liu, and D. G. Truhlar, "Variational transitionstate theory and semiclassical tunnelling calculations with interpolated corrections: a new approach to interfacing electronic structure theory and dynamics for organic reactions," Journal of the Chemical Society Faraday Transactions, vol. 90, 1994.

[22] Y. Kholod, S. Okovytyy, G. Kuramshina, M. Qasim, L. Gorb, and J. Leszczynski, "An analysis of stable forms of CL-20: A DFT study of conformational transitions, infrared and Raman spectra," Journal of Molecular Structure, vol. 843, pp. 14-25, 2007.

[23] B. M. Rice and C. F. Cha Ba Lowski, "Betsy rice," The Journal of Physical Chemistry A, vol. 101, 1997.

[24] K.-B. Sun, S.-H. Zhang, F.-D. Ren, Y.-P. Hao, and S.-H. Ba, "Theoretical prediction of the trigger linkage, cage strain, and explosive sensitivity of CL-20 in the external electric fields," Journal of Molecular Modeling, vol. 27, p. 85, 2021.

[25] Bumpus and A. John, "A Theoretical investigation of the ring strain energy, destabilization energy, and heat of formation of CL-20," Advances in Physical Chemistry, vol. 2012, Article ID 175146, 7 pages, 2014.

[26] L. Lin-Lin and S.-Q. Hu, "Ab initio calculations of the $\mathrm{NO}_{2}$ fission for CL-20 conformers," Journal of Energetic Materials, vol. 37, pp. 154-161, 2019.

[27] T. Zhang, L. Cheng, J. Zhang, and K. Wang, "CPMD investigation of $\alpha$-RDX and $\varepsilon$-CL-20: the transition of deflagration to detonation depending on the self-produced radicals," Physical Chemistry Chemical Physics, vol. 22, pp. 7421-7429, 2020. 